\title{
Decrease of Functioning in Remitted and Non-Remitted Patients 16 Years After a First-Episode Schizophrenia
}

\author{
Carlo Marchesi, MD, * Andrea Affaticati, MD, $†$ Alberto Monici, MD, $\uparrow$ Chiara De Panfilis, $M D, *$ \\ Paolo Ossola, MD, * Rebecca Ottoni, MD, * and Matteo Tonna, $M D{ }^{*}$
}

\begin{abstract}
In schizophrenia, a better level of functioning has been generally associated with symptomatic remission. However, this association has been supported by cross-sectional studies or by studies with a short follow-up period. Forty-eight patients with schizophrenia were evaluated by the Positive and Negative Symptoms Scale and the Social and Occupational Functioning Assessment Scale (SOFAS) at the first episode and after a mean period of 16 years. At follow-up, patients were defined as remitters (R) or non-remitters (NR) according to the Remission Schizophrenia Working Group criteria. $\mathrm{R}$ ( $n=18$; $37.5 \%$ ) compared to NR showed at the first episode a lower illness severity and a better level of functioning. A functional decline was found in both groups at follow-up, even though NR showed a more than twofold reduction than R. Better SOFAS scores at follow-up were predicted by baseline SOFAS score and less severe negative symptoms at follow-up. Schizophrenia implies a functional decline over time, regardless of the symptomatic remission status with negative symptoms playing a major role.
\end{abstract}

Key Words: Schizophrenia, remission, illness severity, positive and negative symptoms, long-term follow-up

(J Nerv Ment Dis 2015;203: 406-411)

A mong the major psychiatric disorders, the longitudinal course and outcome of schizophrenia have been the most extensively studied (Hegarty et al., 1994) because its definition was formerly based, at least in part, on its longitudinal course. Since the description of dementia praecox (Kraepelin, 1899), schizophrenia has been conceptualized as a chronic illness leading to mental deterioration, lack of volition, and social incompetence (Lasser et al., 2007). However, some authors challenged this circular reasoning (because chronic course is used to determine diagnosis, schizophrenia is obviously a chronic illness) (Lieberman et al., 2001) and suggested that outcome in schizophrenia is a complex phenomenon in which the ability of the diagnosis to predict a specific outcome has often been overstated (McGlashan, 2008). Supporting this criticism, many factors have been associated with poor outcome such as poor premorbid adjustment, male gender, younger age at onset, insidious onset, longer interval from the onset to treatment, and severity of symptoms (Andreasen, 2006).

Concerning the premorbid level of functioning, there is general agreement that schizophrenia is characterized by the presence of a premorbid social deficit, i.e., "inadequate pre-psychotic adjustment with little interest in other people or activities of life" (McGlashan, 2008). Such emergence of impaired social competence in the prodromal phase of schizophrenia can impact the subsequent course of the disorder (Gelber et al., 2004; Hafner et al., 1992; Harrow and Jobe, 2007), confirming earlier observations that the level of social maturity reached before the breakdown influences the outcome of a schizophrenic episode (Phillips, 1953).

*Department of Neuroscience, Psychiatry Unit, University of Parma; and $\uparrow$ Mental Health Department, Local Health Agency, Parma, Italy.

Send reprint requests to Matteo Tonna, MD, Department of Neuroscience,

Psychiatry Unit, University of Parma, Ospedale Maggiore, Padiglione Braga,

Viale A. Gramsci 14, 43126 Parma, Italy. E-mail: mtonna@ausl.pr.it. Copyright (C) 2015 Wolters Kluwer Health, Inc. All rights reserved.

ISSN: 0022-3018/15/20306-0406

DOI: 10.1097/NMD.0000000000000299
Remission has long been considered largely impossible in schizophrenia, to the extent that diagnosis and prognosis were viewed as essentially the same (McGlashan, 2008) and a dramatic improvement during the course of the illness was viewed as a sign of earlier misdiagnosis. Recently, however, the Remission in Schizophrenia Working Group (RSWG) (Andreasen et al., 2005) posited that "symptomatic remission is a definable concept and an achievable stage in the treatment of schizophrenia" and proposed criteria for symptomatic remission to support a positive, longer-term approach regarding outcome for patients with schizophrenia. According to these criteria, a relevant proportion of patients (45\% to $70 \%$ ) (Lambert et al., 2010) can be defined as remitters at some point during the course of their illness. Importantly, maintaining low levels of psychopathology may promote productive social and occupational pursuits in these patients (Opler et al., 2007). In support to this view, studies assessing the relationship between remission (defined according to the RSWG criteria) and functional outcome found significantly better levels of functioning in remitted versus nonremitted patients, even though remitted patients showed areas with an inadequate level of functioning (Addington and Addington, 2008; Bobes et al., 2009; Bodén et al., 2009; Karow et al., 2012; Oorschot et al., 2012). These studies confirmed the results of previous investigations using different criteria than those proposed by RSWG (Lasser et al., 2007; Lambert et al., 2008; Lang et al., 2013).

However, studies that applied the RSWG criteria varied substantially in terms of the evaluation time because remission and functioning were assessed cross-sectionally (Oorschot et al., 2012; Helldin et al., 2007), or 2 months after the baseline evaluation (SchennachWolff et al., 2009), 1 to 3 years since the first assessment (Bodén et al., 2009; Addington and Addington, 2009; Chang et al., 2013), or after longer time periods (5-7 years) (Bobes et al., 2009; Ceskova et al., 2011; Kurihara et al., 2011). To our knowledge, no study assessed the association between RSWG-defined remission and level of functioning in patients with schizophrenia after more than 7 years from their first episode of illness. Further, no study investigated if the level of functioning at first admission declines in patients who achieve remission in the course of a long-term follow-up and long duration of illness.

Therefore, the present prospective study assessed patients with schizophrenia both at the time of their first episode and after a mean period of 16 years to examine whether (1) a better level of functioning was associated with the achievement of remission (RSWG criteria) compared to non-remitted patients, (2) remitted patients were characterized by higher levels of functioning at first admission than nonremitters, and (3) the level of functioning at onset deteriorated even in remitted patients, according to a Kraepelinian conceptualization of schizophrenia.

\section{METHODS}

\section{Sample}

The study participants were recruited from patients who were consecutively admitted to the Psychiatry Clinic of the University of Parma, from January 1995 to December 1999, because of a first psychotic episode. To the Psychiatry Clinic were referred patients from 
a population of about 400,000 people. This study is part of a more extensive evaluation of long-term outcome in patients with a first psychotic episode.

Patients were included in the study if (1) they were aged over 17 years; (2) they were admitted for the first time in a psychiatric unit for a first psychotic episode; (3) they were discharged with a diagnosis of Schizophrenia, according to the DSM-IV; and (4) they gave a written informed consent.

Patients were excluded from the study if (1) they were affected by drug abuse or drug dependence, delirium, mental retardation, or organic mental disorders; (2) they were previously treated with antipsychotic medications; and (3) they did not assure treatment adherence or they discontinued the treatment program after discharge from hospital for more than two consecutive visits (see follow-up evaluation). Because the aim of the study was the long-term outcome of patients properly treated for schizophrenia, drug abuse or dependence and nonadherence to treatment were considered exclusion criteria to avoid their confounding effects on outcome. of the study.

The participants gave written consent after complete description

\section{Assessment}

The period of enrollment lasted from January 1995 to December 1999. Patients were evaluated a first time during the index hospitalization and a second time in the year 2010 .

\section{Baseline Assessment}

The baseline evaluation was carried out within the first week of hospitalization. Sociodemographic variables recorded at baseline were age, gender, years of education, marital and employment status, housing situation, age at onset of schizophrenia, and the time passed from the onset of first psychotic symptoms or behavioral changes and hospitalization, where patients received antipsychotic treatment for the first time (see exclusion criteria).

To formulate the diagnosis, we used the information obtained from the Structured Clinical Interview for Axis I DSM-IV disorders, carried out by trained psychiatrists, together with the information collected from family members, medical records, and primary treating physicians.

The severity of symptoms was measured with the Positive and Negative Symptoms Scale (PANSS) (Kay et al., 1987). We used five PANSS scores: positive, negative, general psychopathology, cognitive, and total scores. The cognitive PANSS score was calculated, according to Llorca et al. (2012), by adding the score of the items disorientation (G10), attention deficit (G11), and difficulty in abstract thinking (N5). Consequently, the negative and general psychopathological scores were calculated without the items included in the cognitive score.

Further, according to the PANSS criteria, patients were defined as affected by positive schizophrenia (if they reported a score equal or higher than four in at least three items of positive scale and in less than three items of negative scale), negative schizophrenia (if they reported a score equal or higher than four in at least three items of negative scale and in less than three items of positive scale), or mixed schizophrenia (if they reported a score equal or higher than four in at least three items of positive and negative scales).

The level of functioning was evaluated using the Social and Occupational Functioning Assessment Scale (SOFAS) (APA, 2000). For the attribution of the SOFAS score, we evaluated the habitual way of daily living of each patient in the year before the onset of psychotic symptoms as specified in the instruction for the use of the scale "The SOFAS may also be useful to evaluate functioning for the past year (i.e., the highest level of functioning for at least a few months during the past year)" (page 760) (APA, 2000).

The period of time from the onset of first psychotic symptoms to the hospitalization where patients received treatment for the first time was also calculated.

\section{Follow-Up Evaluation}

After discharged from hospital, patients accessed continuous and reasonably comprehensive public mental health services (Amaddeo et al., 2012), where they attended nearly monthly visits.

During each visit, their psychopathological condition, treatment adherence, medications prescription, and use of illicit drugs were clinically evaluated. The number of psychotic episodes and the number and time of hospitalization were also computed. A psychotic episode (relapse) was defined on the clinical judgment as the reappearance of delusions, hallucinations, and/or behavioral disorganization.

Patients were re-examined in 2010 by a psychiatrist blind to the baseline evaluation. The follow-up assessment consisted in the administration of SCID-IV, PANSS, and SOFAS. After 8 months, the patients were re-evaluated with the PANSS to verify whether the time criterion of 6 months requested for remission, as suggested by RSWG, was satisfied. Patients were defined in remission (R) or in non-remission (NR), according to both severity and time criteria proposed by the RSWG.

\section{Treatment}

All patients were treated with antipsychotic medications. The choice of medication was made on the basis of clinical evaluation and according to the preference of patients and their relatives.

To maximize the treatment adherence, a medication control by relatives was recommended and the count of pills was performed at any time the medication was prescribed. In patients who did not assure a treatment adherence, the use of a long-acting medication was proposed. Patients were considered non-adherent if they reported stopping their antipsychotic for 1 week or more.

Patients also received non-pharmacological treatment such as psycho-education program (together with their families) and social skills and vocational training. In particular, a family psycho-education program was administered only at the beginning of the follow-up period. The program consisted of weekly sessions of 90 minutes for 6 months according to Falloon model (Falloon and Fadden, 1993).

\section{Data Analysis}

Before the start-up of the study and the follow-up evaluations, an inter-rater reliability was calculated for the diagnosis of schizophrenia (baseline: $k=0.92$; follow-up: $k=0.95$ ) for the PANSS total score (baseline: $k=0.82$; follow-up: $k=0.80$ ) and for the SOFAS score (baseline: $k=0.79$; follow-up: $k=0.81$ )

The clinical and sociodemographic features were compared in $\mathrm{R}$ and in NR using the two-tailed Student's $t$ test for continuous variables and the $\chi^{2}$ test for the categorical variables.

The change of symptom severity and SOFAS scores between the baseline and follow-up assessments was evaluated in R and in NR using the two-tailed Student's $t$ test for paired data.

Finally, a multiple regression analysis, stepwise method was used to evaluate the baseline predictors of level of functioning at follow-up. In this analysis, SOFAS score at follow-up was entered as the dependent variable, whereas variables which at baseline showed significant differences between R and NR (baseline SOFAS score, PANSS type, PANSS total score at baseline, number of psychotic episodes and time of hospitalization during follow-up, state of remission, PANSS scores for positive, negative, cognitive, and general psychopathology symptoms at follow-up) (Table 1) were entered as independent variables.

All statistical analyses were performed with SPSS for Windows (version 20; SPSS Inc., Chicago, IL, USA).

\section{RESULTS}

During the period of enrollment, 70 patients were consecutively admitted to the Psychiatric Clinic of the University of Parma for a first 
TABLE 1. Sociodemographic and Clinical Features of Schizophrenic Patients With or Without Remission

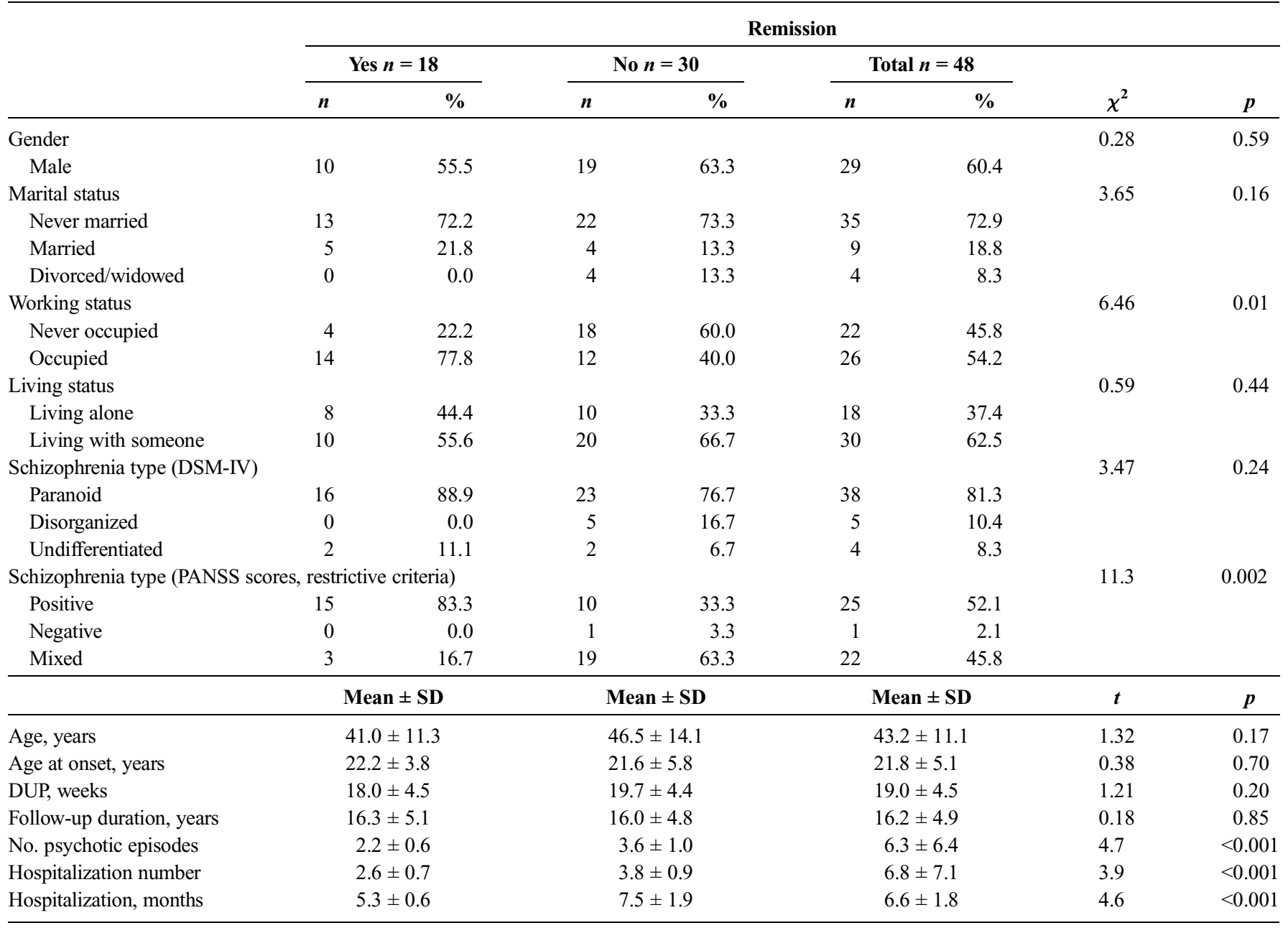

psychotic episode and discharged with a diagnosis of schizophrenia. Among them, 56 accepted to participate in the study.

In 2010, at the time of the follow-up assessment, six patients had moved outside the study area and two patients refused further psychiatric evaluation for the present study. Therefore, only 48 patients were re-evaluated at follow-up.

The SCID-IV re-administration confirmed the stability of schizophrenia diagnosis in all 48 patients, and 18 of them (37.5\%) satisfied remission RSWG criteria (R), whereas the remaining 30 patients $(62.5 \%)$ did not (NR). None of them was using illicit drugs.

\section{Sociodemographic Characteristics}

At first episode, only working status differed between $\mathrm{R}$ and NR patients: $\mathrm{R}$ patients were more likely to be employed than NR patients $\left(\chi^{2}=6.4 ; p=0.01\right)$ (Table 1$)$. The duration of untreated psychosis was short in our sample (Table 1), and no difference was found between $\mathrm{R}$ and NR patients.

At follow-up, duration of illness was similar in $\mathrm{R}$ and in NR patients, whereas the number of psychotic relapses and the number and length of hospitalization during the follow-up period were lower in $\mathrm{R}$ than in NR (Table 1).

\section{Symptom Severity}

A lesser symptom severity at baseline was found in $\mathrm{R}$ than in NR patients: all PANSS dimension scores (positive, negative, cognitive, general psychopathology, and total scores) were lower in R than in NR patients (Table 2).

At follow-up, symptom severity was reduced in both groups of patients as compared to baseline: the decrease reached a statistical significance for PANSS positive, cognitive, general psychopathology, and total scores, but not for negative score, in both R and NR patients (Table 2).

\section{Treatment}

Forty-one patients $(85.5 \%)$ were treated with oral antipsychotics and the remaining seven patients $(14.5 \%)$ received a long-acting antipsychotic injection (LAI). A second-generation antipsychotic (SGA) was administered in 34 patients $(70.8 \%)$ and a first-generation antipsychotic (FGA) was used in 14 patients $(29.2 \%)$. Seven patients switched from an oral FGA to a LAI and 11 patients from a FGA to a SGA. No patient was treated with antipsychotic combination or with electroconvulsive therapy. 
TABLE 2. Symptom Severity (PANSS Scores) and Social and Occupational Functioning Assessment Scale Score in Remitted (R) and in Non-Remitted (NR) Patients

\begin{tabular}{|c|c|c|c|c|c|c|c|c|}
\hline \multirow[b]{4}{*}{ PANSS Symptoms } & \multicolumn{4}{|c|}{ Remission } & \multicolumn{4}{|c|}{$t$ Test for Paired Sample } \\
\hline & \multirow{2}{*}{\multicolumn{2}{|c|}{$\begin{array}{c}\text { Yes } \\
n=18\end{array}$}} & \multirow{2}{*}{\multicolumn{2}{|c|}{$\begin{array}{c}\text { No } \\
n=30\end{array}$}} & \multicolumn{2}{|c|}{ Yes vs. No } & \multicolumn{2}{|c|}{ Baseline vs. Follow-Up } \\
\hline & & & & & \multirow{2}{*}{$\frac{\text { Baseline }}{t}$} & \multirow{2}{*}{$\frac{\text { Follow-Up }}{t}$} & \multirow{2}{*}{$\begin{array}{c}\text { Yes } \\
t\end{array}$} & \multirow{2}{*}{$\begin{array}{c}\text { No } \\
t\end{array}$} \\
\hline & Baseline & Follow-Up & Baseline & Follow-Up & & & & \\
\hline Positive & $24.8 \pm 3.7$ & $11.0 \pm 2.7$ & $28.1 \pm 4.0$ & $17.2 \pm 5.8$ & $2.7 * *$ & $4.2 * *$ & $13.9^{* *}$ & $11.5^{* *}$ \\
\hline Negative & $15.6 \pm 2.6$ & $14.3 \pm 2.6$ & $22.0 \pm 3.7$ & $21.1 \pm 4.1$ & $6.3^{* *}$ & $6.2 * *$ & 1.8 & 1.4 \\
\hline General & $42.1 \pm 3.6$ & $29.4 \pm 5.2$ & $50.4 \pm 4.9$ & $37.7 \pm 6.3$ & $6.6^{* *}$ & $4.7^{* *}$ & $8.9^{* *}$ & $12.7 * *$ \\
\hline Cognitive & $6.1 \pm 2.1$ & $5.2 \pm 1.5$ & $8.6 \pm 1.9$ & $6.9 \pm 2.4$ & $4.3^{* *}$ & $2.5^{*}$ & 2.0 & $4.7 * *$ \\
\hline Total score & $88.8 \pm 6.2$ & $60.7 \pm 10.5$ & $109 \pm 10.0$ & $83.0 \pm 14.2$ & $7.7 * *$ & $4.6^{* *}$ & $10.5^{* *}$ & $12.2^{* *}$ \\
\hline SOFAS score & $67.7 \pm 7.3$ & $62.2 \pm 7.3$ & $58.0 \pm 8.7$ & $45.3 \pm 10.6$ & $6.1 * *$ & $5.9 * *$ & $10.0^{* *}$ & $8.4 * *$ \\
\hline
\end{tabular}

PANSS Negative: all negative symptoms except difficulty in abstract thinking score. PANSS General: all general psychopathological symptoms except disorientation and poor attention. PANSS Cognitive: disorientation, poor attention and difficulty in abstract thinking.

$* p<0.05 ; * p<0.001$.

\section{Level of Functioning}

At baseline, the SOFAS score was higher in R than in NR patients (Table 2). At follow-up, a significant decrease in SOFAS score was observed in both $\mathrm{R}$ and NR patients (Table 2), but this decrease was smaller in $\mathrm{R}(5.5 \pm 2.3)$ than in NR patients $(12.6 \pm 8.1)(t=3.4$; $d f=46 ; p=0.001$ ) (Fig. 1).

In the multiple regression analysis, the SOFAS score at baseline and the PANSS negative score at follow-up predicted the SOFAS score at follow-up, whereas no effect was observed for PANSS type, baseline PANSS total score, number of psychotic episodes and time of hospitalization during follow-up, PANSS positive, cognitive, and psychopathological general scores at follow-up, and the remission status (Table 3). This regression model explained $77 \%$ of variance $\left(R^{2}\right)$ of the SOFAS score at follow-up.

\section{DISCUSSION}

The present study aimed at evaluating the long-term course and the potential correlates of functional impairment in a sample of 48 reliably diagnosed patients with schizophrenia. The level of psychosocial

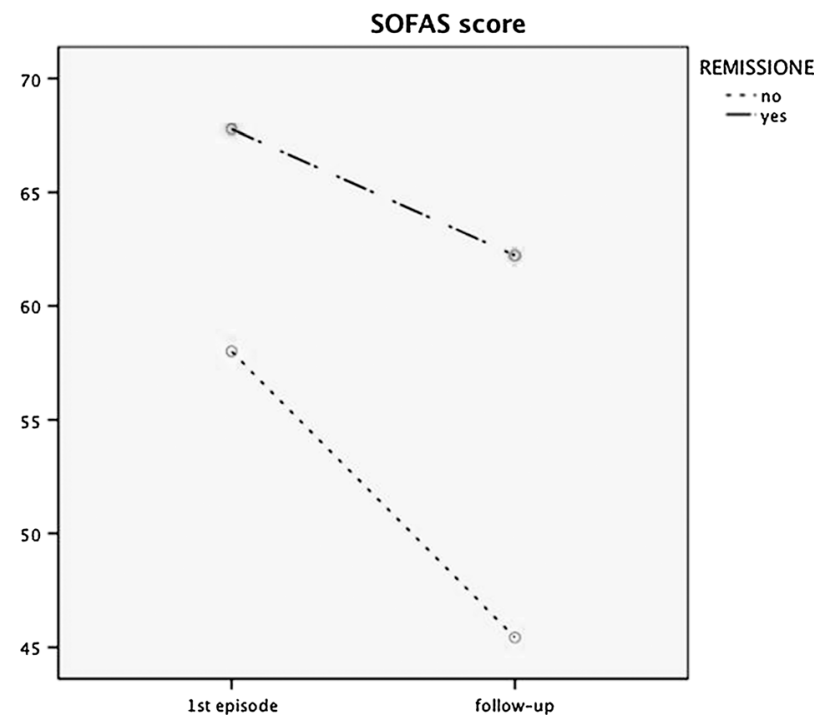

FIGURE 1. SOFAS score in remitted and in non-remitted schizophrenic patients in the year before the first episode and at follow-up. functioning as well as the severity of symptoms was assessed both at the first episode and after a mean period of 16 years of illness.

At follow-up, 18 patients (37.5\%) (R) satisfied the remission criteria (both severity and time criteria) proposed by the RSWG (Andreasen et al., 2005), whereas the remaining 30 patients did not reach remission $(\mathrm{NR})$.

\section{Remitted Versus Non-Remitted Patients at First Episode and Follow-Up}

Remitters and non-remitters showed clinical differences at their first episode of illness: $\mathrm{R}$ patients had a lower severity of symptoms and a better level of functioning in the year before the first admission than NR. After 16 years of illness, an improvement in PANSS positive, cognitive, and general psychopathological symptoms was found in both $\mathrm{R}$ and NR, whereas no change in severity of negative symptoms was observed in the two groups. In contrast, a decrease of functioning was observed regardless of the achievement of the symptomatic remission, although a more than twofold reduction of SOFAS scores was found in NR than in R.

Therefore, our findings suggest that remission of symptoms, although associated with a better functioning, do not completely prevent the worsening of social abilities, but may mitigate the functional decline if compared to non-remission.

On the one hand, these findings are in keeping with several previous studies (Addington and Addington, 2008; Bobes et al., 2009;

TABLE 3. Baseline Predictors of the SOFAS Score at Follow-Up (Stepwise Multiple Regression Analysis)

\begin{tabular}{lcrcc}
\hline SOFAS Score at Follow-Up & $\boldsymbol{b}$ & $\boldsymbol{t}$ & $\boldsymbol{p}$ & $\boldsymbol{R}^{\mathbf{2}}$ \\
\hline Step 1 & & & & 0.65 \\
$\quad$ SOFAS score at baseline & 0.80 & 9.2 & $<0.001$ & \\
$\quad$ Step 2 & & & & 0.77 \\
$\quad$ SOFAS score at baseline & 0.49 & 5.2 & $<0.001$ & \\
$\quad \begin{array}{l}\text { PANSS Negative symptoms score } \\
\quad-0.47\end{array}$ & -4.9 & $<0.001$ & \\
$\quad$ at follow-up & & & &
\end{tabular}

In the multiple regression analysis, PANSS type, baseline PANSS total score, baseline SOFAS score, follow-up PANSS positive, negative, cognitive, and general psychopathological symptoms scores, number of psychotic episodes, time of hospitalization, and remission status $(0=$ no; $1=$ yes $)$ entered as independent variables. 
Bodén et al., 2009; Karow et al., 2012; Oorschot et al., 2012), which demonstrated that remission of symptoms was associated with a better level of functioning. On the other hand, they suggest that the decline in psychosocial functioning seems to be inherently associated with the course of the illness because a decrease in social abilities was also observed in $\mathrm{R}$ patients. Consistently, the regression analysis demonstrated that symptomatic remission did not predict a better functional outcome at follow-up.

\section{Symptom Severity and Social Functioning}

A possible interpretation of these results is that low-mild symptoms, which do not significantly interfere with behavior, may let the $\mathrm{R}$ individual to develop coping mechanisms and to better respond to psychosocial therapies and rehabilitation interventions. In contrast, more severe symptoms may negatively influence the levels of functioning, so as to explain a greater deterioration in social functions in NR than in R patients. Nevertheless, overall social functioning appears to worsen over time in both $\mathrm{R}$ and NR patients. This means that a greater symptom severity cannot justify alone the poorer functional outcome in NR. In fact, NR showed a greater deficit of functioning already at the onset of the illness and a greater functional impairment over the course of follow-up in opposition to the improvement of most of their symptoms (i.e., positive, general psychopathology, and cognitive).

Interestingly, an inverse relationship between negative symptoms severity and functional outcome was observed because a higher PANSS negative score at follow-up was related to a lower SOFAS score at the end of the study, thus confirming the results of previous longterm studies (Bobes et al., 2009; Ceskova et al., 2011; White et al., 2009). In the present study, the severity of negative symptoms appeared stable over time, which seems in contrast with the decline in functioning over time. This might confirm that functional impairment is only partly dependent from symptom severity and that other mechanisms, i.e., cognitive dysfunctions and premorbid functioning (Lieberman et al., 2001; Bowie et al., 2008; Walker, 1994), might be involved in the decrease of social functioning. Nevertheless, it cannot be excluded that negative symptoms might exert a continuous and cumulative effect on functioning, which was maintained independently from an increase in their severity.

\section{Premorbid Functioning and Outcome}

As a further suggestion that other factors besides symptom dimensions may be involved in the deterioration of levels of functionality, we found that the poor SOFAS score at baseline was the major predictor of SOFAS score at follow-up. Thus, our findings confirm the longstanding observation that social abnormalities are already present at the onset of illness and that poorer adjustment at the onset is associated with a decline of social functioning (Lieberman et al., 2001; Chang et al., 2013; Ayesa-Arriola et al., 2013). A poor premorbid adjustment has been postulated to be indicative of a more deteriorating form of illness, and a poor adjustment in the year before the first admission (a SOFAS score lower than 70) was observed in $91.7 \%$ of our patients. This finding may explain why in the current study a worsening of functioning was found in all of our patients regardless of their clinical improvement

The relationship between first-episode adjustment and negative symptoms is complex and difficult to disentangle. Interestingly, previous studies suggested that premorbid maladjustment, and particularly impairment in social functioning, may represent a precursor of negative symptoms (Chang et al., 2013). Therefore, we may hypothesize that in our patients a poor social functioning at first admission may facilitate the onset of negative symptoms, which in turn contributes to the further deterioration of social functions.

\section{Limitations}

The present study has the following limitations.
First, symptom severity was evaluated with PANSS only at onset and after a mean period of 16 years of illness, raising the question of how representative the last 6 months of illness are for the total duration of illness. However, it is well known that the remission status could not be a stable condition, and even though in the present study patients in remission had relapses, they showed a less number of relapses than in non-remitters, suggesting a more favorable course of illness in remitters than in non-remitters.

Second, we evaluated social functioning only with a unidimensional instrument (SOFAS) instead of using multivariate measures. Therefore, different areas of social and daily functioning could not be specifically assessed to verify which area of long-term social functions was impaired in our patients.

Third, we evaluated cognitive symptoms only by the means of the PANSS items "disorientation, attention deficit and difficulty in abstract thinking". Therefore, a more comprehensive cognitive evaluation is warranted before concluding that cognitive deficit does not impact on long-term functioning, as we found in the present study. However, recent studies found that neurocognition deficits did not predict long-term functional outcome (Ayesa-Arriola et al., 2013).

Finally, the current study includes patients who were treated a short time after the onset of psychotic symptoms, who were strictly treatment adherent and substance use free. Thus, the results of the study are not applicable to overall schizophrenia patients.

\section{CONCLUSIONS}

In conclusion, the present study indicates that, first, symptomatic remission is not necessarily associated with functional improvement because symptoms and social functioning do not follow a parallel course; second, the level of functionality in the year before the first admission is the major predictor of the functional outcome after many years of illness, explaining most (65\%) of the variance of SOFAS score at the end of the study; third, negative symptoms, associated with poor social functioning in the year before the first admission, exert a cumulative effect on the decline of social functions.

\section{DISCLOSURES}

No authors received funding for the research and had financial involvement that could represent potential conflicts of interest.

\section{REFERENCES}

Addington J, Addington D (2008) Symptom remission in first episode patients. Schizophr Res. 106:281-285.

Addington J, Addington D (2009) Three-year outcome of treatment in early psychosis program. Can J Psychiatry. 54:626-630.

Amaddeo F, Barbui C, Tansella M (2012) State of psychiatry in Italy 35 years after psychiatric reform. A critical appraisal of national and local data. Int Rev Psychiatry. 24:314-320

American Psychiatric Association (2000) Diagnostic and Statistical Manual of Mental Disorders. 4th ed Washington DC: American Psychiatric Association.

Andreasen NC (2006) Standardized remission criteria in schizophrenia. Acta Psychiatr Scand. 113:81.

Andreasen NC, Carpenter WTJR, Kane JM, Lasser RA, Marder SR, Weinberger DR (2005) Remission in schizophrenia: Proposed criteria and rationale for consensus. Am J Psychiatry. 162:441-449.

Ayesa-Arriola R, Manuel Rodríguez-Sánchez J, Pérez-Iglesias R, González-Blanch C, Pardo-García G, Tabares-Seisdedos R, Vazquez-Barquero JL, Crespo-Facorro B (2013) The relevance of cognitive, clinical and premorbid variables in predicting functional outcome for individuals with first-episode psychosis: A 3 year longitudinal study. Psychiatry Res. 209:302-308.

Bobes J, Ciudad A, Alvarez E, San L, Polavieja P, Gilaberte I (2009) Recovery from schizophrenia: Results from a 1-year follow-up observational study of patients in symptomatic remission. Schizophr Res. 115:58-66. 
Bodén R, Sundström J, Lindström E, Lindström L (2009) Association between symptomatic remission and functional outcome in first-episode schizophrenia. Schizophr Res. 107:232-237.

Bowie CR, Leung WW, Reichenberg A, McClure MM, Patterson TL, Heaton RK, Harvey PD (2008) Predicting schizophrenia patients' real-world behavior with specific neuropsychological and functional capacity measures. Biol Psychiatry. 63:505-511.

Ceskova E, Prikryl R, Kasparek T (2011) Outcome in males with first-episode schizophrenia: 7-year follow-up. World J Biol Psychiatry. 12:66-72.

Chang WC, Yee Man Tang J, Lai Ming Hui C, Hoi Yan Wong G, Kit Wa Chan S, Ho Ming Lee EH, Chen EY (2013) The relationship of early premorbid adjustment with negative symptoms and cognitive functions in first-episode schizophrenia: A prospective three-year follow-up study. Psychiatry Res. 209:353-360.

Falloon IRH, Fadden G (1993) Integrated Mental Health Care: A Comprehensive, Community-Based Approach. Cambridge: Cambridge University Press.

Gelber EI, Kohler CG, Bilker WB, Gur RC, Brensinger C, Siegel SJ, Gur RE (2004) Symptom and demographic profiles in first-episode schizophrenia. Schizophr Res. 67:185-194.

Hafner H, Riecher-Rössler A, Hambrecht M, Maurer K, Meissner S, Schmidtke A, Fatkenheuer B, Loffler W, van der Heiden W (1992) IRAOS: An instrument for the assessment of onset and early course of schizophrenia. Schizophr Res. 6:209-223.

Harrow M, Jobe TH (2007) Factors involved in outcome and recovery in schizophrenia patients not on antipsychotic medications: A 15-year multifollow-up study. J Nerv Ment Dis. 195:406-414.

Hegarty JD, Baldessarini RJ, Tohen M, Waternaux C, Oepen G (1994) One hundred years of schizophrenia: A meta-analysis of the outcome literature. Am J Psychiatry. 151:1409-1416.

Helldin L, Kane JM, Karilampi U, Norlander T, Archer T (2007) Remission in prognosis of functional outcome: A new dimension in the treatment of patients with psychotic disorders. Schizophr Res. 93:160-168.

Karow A, Moritz S, Lambert M, Schöttle D, Naber D (2012) Remitted but still impaired? Symptomatic versus functional remission in patients with schizophrenia. Eur Psychiatry. 27:401-405.

Kay SR, Fiszbein A, Opler LA (1987) The positive and negative syndrome scale (PANSS) for schizophrenia. Schizophr Bull. 13:261-276.

Kraepelin E (1899) Ein Lehrbuch für Studierende und Äertze. 6. Auflage. Psychiatrie. Leipzig, Austria: Johann Ambrosins Barth.

Kurihara T, Kato M, Reverger R, Tirta IGR (2011) Remission in schizophrenia: A community-based 6-year follow-up study in Bali. Psychiatry Clin Neurosci. $65: 476-482$
Lambert M, De Marinis T, Pfeil J, Naber D, Schreiner A (2010) Establishing remission and good clinical functioning in schizophrenia: Predictors of best outcome with long-term risperidone long-acting injectable treatment. Eur Psychiatry. 25:220-229.

Lambert M, Naber D, Schacht A, Wagner T, Hundemer H-P, Karow A, Huber CG, Suarez D, Haro JM, Novick D, Dittmann RW, Schimmelmann BG (2008) Rates and predictors of remission and recovery during 3 years in 392 never-treated patients with schizophrenia. Acta Psychiatr Scand. 118:220-229.

Lang FU, Kösters M, Lang S, Becker T, Jäger M (2013) Psychopathological long-term outcome of schizophrenia - a review. Acta Psychiatr Scand. 127:173-182.

Lasser RA, Nasrallah H, Helldin L, Peuskens J, Kane J, Docherty J, Tronco AT (2007) Remission in schizophrenia: Applying recent consensus criteria to refine the concept. Schizophr Res. 96:223-231.

Lieberman JA, Perkins D, Belger A, Chakos M, Jarskog F, Boteva K, Gilmore J (2001) The early stages of schizophrenia: Speculations on pathogenesis, pathophysiology, and therapeutic approaches. Biol Psychiatry. 50:884-897.

Llorca P-M, Blanc O, Samalin L, Bosia M, Cavallaro R (2012) Factors involved in the level of functioning of patients with schizophrenia according to latent variable modeling. Eur Psychiatry. 27:396-400.

McGlashan TH (2008) Premorbid adjustment, onset types, and prognostic scaling: Still informative? Schizophr Bull. 34:801-805.

Oorschot M, Lataster T, Thewissen V, Lardinois M, van Os J, Delespaul PA, MyinGermeys I (2012) Symptomatic remission in psychosis and real-life functioning. Br J Psychiatry. 201:215-220.

Opler MG, Yang LH, Caleo S, Alberti P (2007) Statistical validation of the criteria for symptom remission in schizophrenia: Preliminary findings. BMC Psychiatry. $7: 35$.

Phillips L (1953) Case history data and prognosis in schizophrenia. J Nerv Ment Dis. 117:515-525.

Schennach-Wolff R, Jäger M, Seemüller F, Obermeier M, Messer T, Laux G, Pfeiffer H, Naber D, Schmidt LG, Gaebel W, Huff W, Heuser L, Maier W, Lemke MR, Ruther E, Buchkremer G, Gastpar M, Moller HJ, Riedel M (2009) Defining and predicting functional outcome in schizophrenia and schizophrenia spectrum disorders. Schizophr Res. 113:210-217.

Walker EF (1994) Developmentally moderated expressions of the neuropathology underlying schizophrenia. Schizophr Bull. 20:453-480.

White C, Stirling J, Hopkins R, Morris J, Montague L, Tantam D, Lewis S (2009) Predictors of 10-year outcome of first-episode psychosis. Psychol Med. 39: 1447-1456. 